\title{
ELECTRO-MAGNETO-FLUID-STRUCTURAL COUPLING PROBLEM: THE VIBRATING VISCOMETER
}

\author{
Doudou Badiane* \\ PRISME Laboratory \\ University of Orléans \\ Orléans, France 45000
}

Email: doudou.badiane@univ-orleans.fr

\author{
Alain Gasser \\ alain.gasser@univ-orleans.fr \\ Eric Blond \\ eric.blond@univ-orleans.fr
}

\author{
Luc Bellière \\ Kevin Vancayzeele \\ SOFRASER \\ Villemandeur, France
}

Email: instruments@sofraser.com

\begin{abstract}
This study presents a viscosity sensor that converts the vibration amplitude at the resonance frequency of a needle immersed in viscous fluid, into electric current. The goal of this work is to provide a dedicated numerical tool for the sensor design, that couples fluid, structure and magnetism.
\end{abstract}

\section{NOMENCLATURE}

$Q \quad$ Quality factor.

$\omega \quad$ Angular frequency.

$R \quad$ Radius of the needle.

$X \quad$ Vibration amplitude.

$k_{m} \quad$ Added mass.

$k_{d} \quad$ Damping coefficient.

$K_{0}, K_{1} \quad$ Modified Bessel functions of second kind.

$R_{e} \quad$ Reynolds number.

$\rho \quad$ Fluid density.

$R \quad$ Radius of the beam.

$\mu \quad$ Kinematic viscosity.

$\wedge \quad$ Hydrodynamic function.

$\Gamma \quad$ Hydrodynamic coefficient.

$F \quad$ Hydrodynamic force.

$\vec{A} \quad$ Magnetic potentiel vector.

$\vec{H} \quad$ Magnetic field.

$\vec{B} \quad$ Magnetic flux density.

$\vec{E} \quad$ Electric field.

$\vec{D} \quad$ Electric displacement field.

$\sigma \quad$ Electric conductivity.

$T_{2} \quad$ Stress tensor of air.

$\overrightarrow{J_{e}} \quad$ External current density.

*Address all correspondence to this author.

$\begin{array}{ll}\vec{n} & \text { Normal pointing out. } \\ \vec{V} & \text { Velocity. } \\ \varsigma & \text { Charge density. } \\ \mu_{r} & \text { Relative permeability. } \\ \mu_{0} & \text { Vacuum permeability. }\end{array}$

\section{INTRODUCTION}

Viscosity sensors using piezoelectric material and magnetostrictive material have been developed to be used in oil, food or cosmetic industries to control on-line processes [1]. However they require high-frequency system. Cantilever structures commonly used in atomic force microscopy [2] as mass-sensitive devices [3], and biosensors [4], in both liquid and gaseous environments are used increasingly in applications involving the viscosity sensing. In this work, a numerical model of a viscometer based on cantilever structures (Fig. 1), including all the physics (magnetism, vibration, fluid mechanics) is proposed. The vibration of the oscillator is due to an electric current through a coil which is under a static magnetic field. When the mechanical oscillator is immersed in a viscous fluid, the vibration amplitude decreases because of the energy dissipation. In these conditions, the quality factor $Q$ that is the ratio of the energy stored in the oscillator to the energy supplied can be significantly reduced. Thereby the goal of this work is the modeling and the semi numerical computation of a fluid-structure-magnetic interaction problem, where commercial finite element programs show huge difficulties in an adequate resolution of fluid, beam and magnetic dynamics due to computational limitations. 


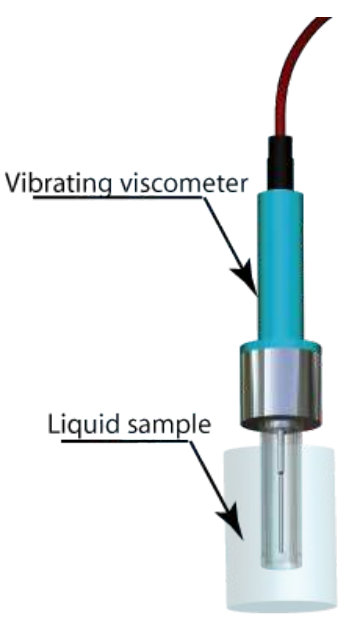

FIGURE 1. VIBRATING VISCOMETER.

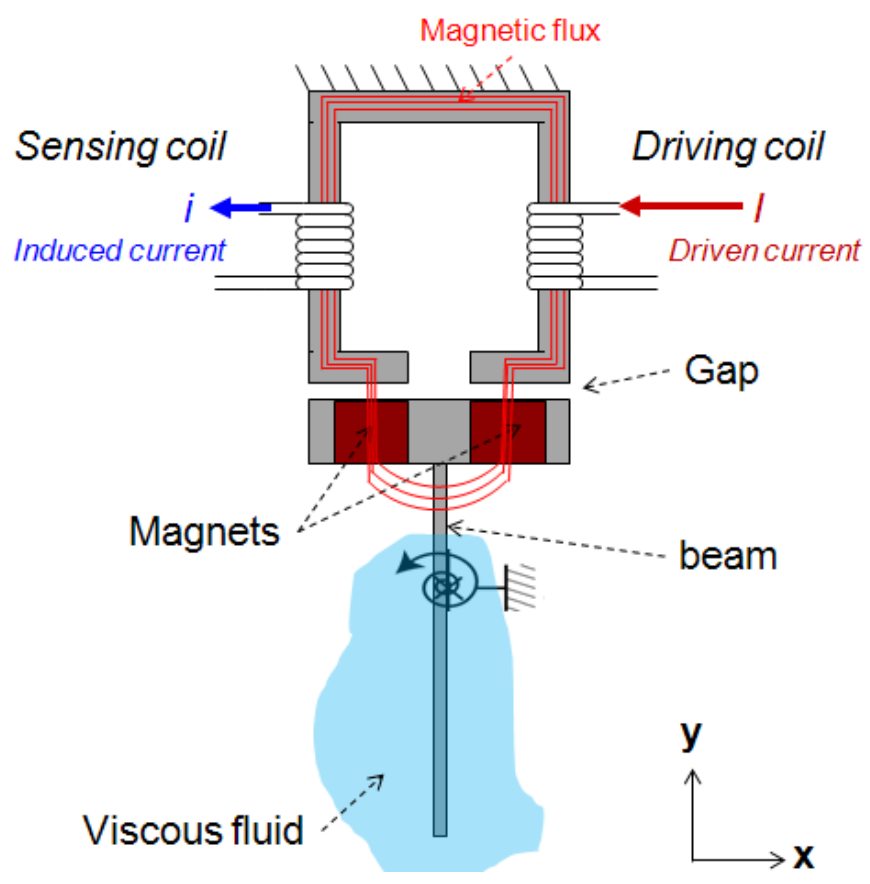

FIGURE 2. Schematic view of the sensor.

\section{METHODS AND MATERIALS}

Fig. 2 shows schematic view of the sensor. It is composed of a mechanical oscillator, permanent magnets and two coils. A driven alternative current at fixed frequency is applied through the first coil as a vibration source for the oscillator. This vibration generates induced current in the second coil, that is proportional to the vibration amplitude and therefore to fluid viscosity. In this study, Bernoulli-Euler equations are used to model the needle. The action of the surrounding fluid is replaced by the hydrodynamic resistance coefficients reflecting the added mass and damping coefficients proposed by Tuck [5]. The excitation system is modeled as magneto-structure interaction using Maxwell equations.

\section{Fluid-structure interaction}

In case of small vibration, the hydrodynamic force is a function of the vibration amplitude meaning that

$$
F=\wedge(\omega) X(x, \omega)
$$

in time harmonic terms $[5,6]$ where $X$ is the vibration amplitude and $\omega$ the angular frequency. Tuck has shown that [5]:

$$
\wedge(\omega)=\left(k_{m}-j k_{d}\right) \pi \rho R^{2} \omega^{2}
$$

where $k_{m}$ is the added mass coefficient, $k_{d}$ the damping coefficient, $R$ the radius of the beam. In these conditions the Reynolds number is:

$$
R_{e}=\frac{\rho \omega D^{2}}{4 \mu}
$$

where $\rho$ is the density of the fluid, $D$ the diameter of the beam, $\omega$ the angular frequency and $\mu$ the kinematic viscosity. Stokes [7] provided a solution to Tuck's formulation:

$$
k_{m}-j k_{d}=1+\frac{4 i K_{1}\left(-i \sqrt{i R_{e}}\right)}{\sqrt{i R_{e}} K_{0}\left(-i \sqrt{i R_{e}}\right)}
$$

where $K_{0}, K_{1}$ are modified Bessel functions of second kind,

$$
\Gamma(\omega)=k_{m}-j k_{d}
$$

is a hydrodynamic coefficient.

\section{Magneto-structural interaction}

In this paper, all the numerical computations are performed using the multiphysic coupling code COMSOL [8]. Many environnements modeled with finite elements are unbounded or open, meaning that the electromagnetic fields extend toward infinity (see Fig. 3). Several approaches are used to model unbounded domains [9]. Here implementation of infinite element approach is used and the elements are referred to mapped infinite elements [10]. This implementation maps the model coordinates from the local, finite-sized domain to a stretched domain.

Under the quasi-static approximation, the electromagnetic problem on macroscopic level consists of solving Maxwell's equations. These equations for general time-varying fields in terms of 
magnetic potentiel vector can be written as

$$
\left\{\begin{array}{l}
\sigma \frac{\partial \vec{A}}{\partial t}+\nabla \times\left(\mu_{0}^{-1} \mu_{r}^{-1} \vec{B}\right)+\sigma(\vec{V} \times \vec{B})=\vec{J}_{e} \\
\vec{B}=\nabla \times \vec{A} \\
\vec{B}=\mu_{0} \mu_{r} \vec{H}
\end{array}\right.
$$

for Ampere's law while Faraday's Law is

$$
\left\{\begin{array}{l}
\nabla \times \vec{E}=-\frac{\partial \vec{B}}{\partial t} \\
\nabla \cdot \vec{D}=\varsigma
\end{array}\right.
$$

where $\sigma$ is the electric conductivity, $\varsigma$ is a space charge density, $\mu_{0}$ and $\mu_{r}$ are respectively vacuum and relative permeability, $\vec{A}$ is the magnetic potentiel vector, $\vec{B}$ is the magnetic field, $\vec{V}$ represents the velocity of the moving geometries, $\vec{J}_{e}$ is generated externally current density, $\vec{E}$ is the electric field and $\vec{D}$ the electric displacement field.

When a structure is surrounded by air in Fig. 4, the stress tensor must be continuous. This gives rise to a surface force acting on the boundary between the two materials. In this case, the air stress tensor is

$$
T_{2}=-p I-\frac{1}{2}(\vec{E} \cdot \vec{D}+\vec{H} \cdot \vec{B}) I+\vec{E} \vec{D}^{t}+\vec{H} \vec{B}^{t}
$$

where $p$ is the air pressure, $I$ is the identity $3 \times 3$ tensor, and $\vec{B}$ and $\vec{H}$ are $3 \times 1$ vectors.

When air is approximated by vacuum, $p=0$ and the equation (8) with $p=0$ is known as the Maxwell stress tensor. In these conditions, the vibration source force acting on the moving geometries linked to the needle is computed by projection of the stress tensor on the outside of these solid surfaces.

$$
\vec{n} T_{2}=-p \vec{n}-\frac{1}{2}(\vec{E} \cdot \vec{D}+\vec{H} \cdot \vec{B}) \vec{n}+(\vec{n} \cdot \vec{E}) \vec{D}^{t}+(\vec{n} \cdot \vec{H}) \vec{B}^{t}
$$

where $\vec{n}$ is the boundary normal pointing out from the needle, $T_{2}$ is the stress tensor of air, $\vec{H}$ is the magnetic field and $\vec{B}$ the magnetic flux density.

\section{RESULTS}

In the present work, different viscous fluids were studied. As shown in Fig. 5 the vibration amplitude is decreased with the increase of the fluid viscosity. The driven current is imposed to investigate the effect of vibration amplitude on the induced current. The sensing current around the second coil and the vibration amplitude at the free-end of the needle are increased/decreased depending on the fluid viscosity surrounding the beam (Fig. 6).

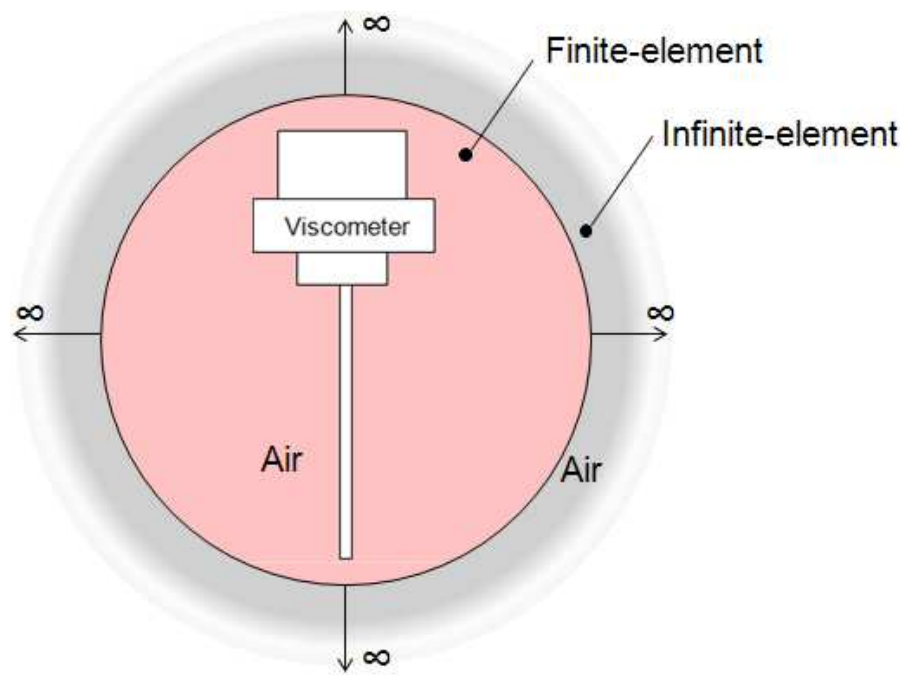

FIGURE 3. SENSOR SURROUNDED BY AIR DOMAINE.

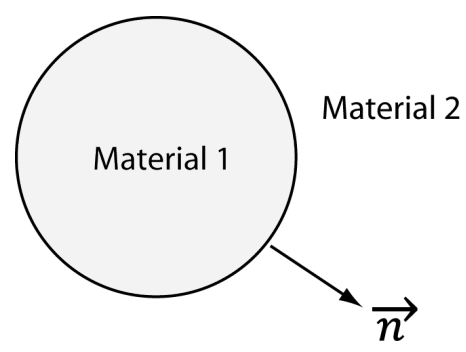

FIGURE 4. SOLID MATERIAL SURROUNDED BY AIR.

\section{CONCLUSION AND PROSPECTS}

The numerical vibrating viscosity sensor is developed for investigation of newtonian fluid viscosity. The output signals obtained from the sensing coil are proportional to the vibration amplitude. Obtained results are validated by experiments on the existing viscometer. A parametric study of the viscometer is now being performed to better understand the sensor behavior for its miniaturization.

\section{ACKNOWLEDGMENT}

This work is funded by the General Council of Loiret (France) and SOFRASER company.

\section{REFERENCES}

[1] Pao, R. H. F., 1961. Fluid Mechanics. Wiley, New York.

[2] Sader, J. E., 1998. "Frequency response of cantilever beams immersed in viscous fluids with applications to the atomic 


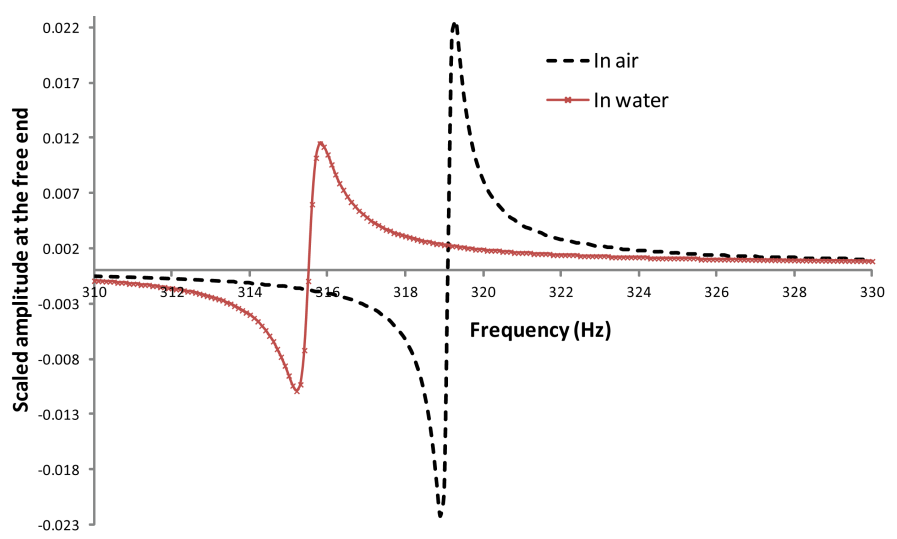

FIGURE 5. FREQUENCY RESPONSE OF THE VISCOMETER.

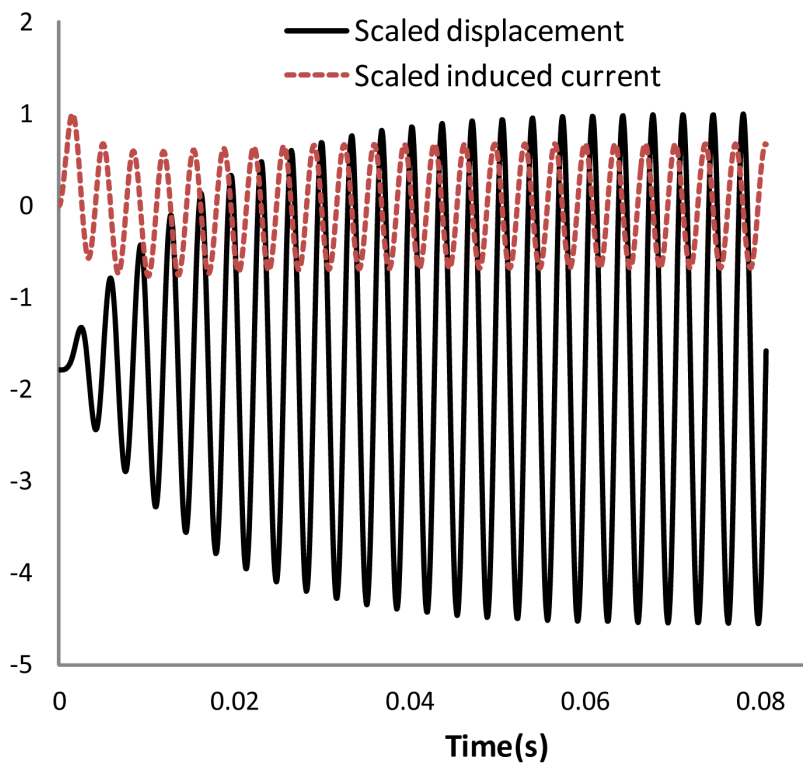

FIGURE 6. VIBRATION AMPLITUDE AND SENSING CURRENT.

force microscope". Journal of Applied Physics, 84(1), pp. 64-76.

[3] Villarroya, M., Verd, J., and Teva, J., 2005. "Cantilever based mems for multiple mass sensing". Res. Microelectron Electron., 1, pp. 197-200.

[4] Vancura, C., Li, Y., and Josse, F., 2005. "Fully integrated cos resonance cantilever sensor for biochemical detection in liquid environments". Transducers05, Digest of Tech, 1, pp. 1142-1145.

[5] Tuck, E. O., 1969. "Calculation of unsteady flows due to small motions of cylinders in a viscous fluid". Journal of Engineering Mathematics, 3(1), pp. 29-44.
[6] Weiss, B., Reichel, E. K., and Jakoby, B., 2008. "Modeling of a clamped-clamped beam vibrating in a fluid for viscosity and density sensing regarding compressibility". Sensors and Actuators A: Physical, 143(2), pp. 293 - 301.

[7] Rosenhead, L., 1963. "Laminar boundary layers". Clarendon, Oxford.

[8] COMSOL, 2011. Comsol multiphysics user guide.

[9] Houmat, A., 2008. "Mapped infinite p-element for twodimensional problems of unbounded domains". Computers and Geotechnics, 35(4), pp. 608-615.

[10] Zienkiewicz, I. O. C., Emson, C., and Bettess, P., 1983. "A novel boundary infinite element". International Journal for Numerical Methods in Engineering, 19, pp. 393-404. 